\title{
Curvas de velocidade da altura e os parâmetros do salto pubertário de crianças e adolescentes Caririenses
}

http://dx.doi.org/10.11606/1807-5509201700040729

\author{
Simonete SILVA* \\ Duarte FREITAS ${ }^{* *}$ \\ José MAIA***
}

${ }^{*}$ Centro de Ciências

Biológicas e da

Saúde, Universidade

Regional do Cariri,

Crato, CE, Brasil.

**Faculdade de

Ciências Sociais,

Universidade da

Madeira, Funchal,

Portugal.

***Faculdade

de Desporto,

Universidade do

Porto, Porto, Portugal.

\section{Resumo}

Apresentamos as curvas médias da distância e velocidade da altura, as idades em que ocorreram os parâmetros do salto pubertário e comparamos os valores obtidos com referências nacionais e internacionais. A amostra contém 280 crianças e adolescentes dos dois sexos que participaram do Projeto Crescer com Saúde no Cariri. Foi utilizado o modelo logístico triplo implementado no software AUXAL. As idades no take-off ocorreram aos 8.8 e 10.3 anos, a altura alcançada foi de 127.3 e $134.0 \mathrm{~cm}$ e a velocidade foi de $4.7 \mathrm{~cm} /$ ano para ambos os sexos. 0 pico de velocidade da altura (PVA) ocorreu aos 11.6 anos nas meninas e aos 13.6 anos nos meninos. A altura média alcançada foi de 143.5 e $154.8 \mathrm{~cm}$ com uma velocidade de 7.1 e $8.7 \mathrm{~cm} /$ ano entre meninas e meninos, respectivamente. As curvas de crescimento da altura das crianças do Cariri mostram um padrão semelhante ao observado em outros estudos. Apesar da defasagem temporal entre o presente estudo e os demais utilizados nas comparações, as idades de ocorrência do PVA, bem como os valores encontrados nos parâmetros do salto pubertário são semelhantes.

PalavRAS-chave: Crescimento; Maturação; Crianças; Adolescentes; Altura.

\section{Introdução}

É inquestionável a presença de enorme variação nos valores da altura das populaçôes modernas ${ }^{1}$, ainda que as explicaçôes deste fato nem sempre sejam consensuais ${ }^{2,3}$. A monitorização da altura é corrente e de grande utilidade na Pediatria ${ }^{4}$ e em Saúde Pública ${ }^{5}$, bem como nos estudos em Educação Física e Esporte com crianças e jovens ${ }^{6-10}$. Neste último domínio é matéria de interesse face ao modo como se interpreta o desempenho motor quando os dados se alinham pela idade em que ocorre o pico de velocidade da altura (PVA) ${ }^{11,12}$.

A informaçáo de natureza transversal sobre os valores da altura de um qualquer grupo populacional permite construir cartas percentílicas da distância do crescimento, ou seja, representar graficamente numa carta os seus valores de referência de acordo com a idade e sexo - habitualmente do percentil 3 ao percentil $97^{13}$. O tipo de curvas produzidas por esta espécie de dados fornece uma imagem estática da variaçáo da altura em cada idade, por exemplo entre 2 e os 20 anos $^{14}$. Em contrapartida, a pesquisa sobre a velocidade do crescimento da altura permite identificar diferenças que ocorrem em distintas características biológicas que a descrevem, i.e., nos parâmetros do salto pré-pubertário e pubertário, ao mesmo tempo que informa sobre o seu timing - momento em que um dado evento maturacional ocorre, e tempo - ritmo com que esse evento se manifesta ${ }^{15}$. A adequada interpretação da dinâmica, ou cinética, do crescimento da altura exige informação de natureza longitudinal ou longitudinal-mista ${ }^{16}$.

Ainda que menos frequentes do que as curvas da distância da altura, ou de qualquer outro 
indicador do crescimento físico humano, as curvas de velocidade são relativamente escassas porque os estudos longitudinais-puros disponíveis não são abundantes, sendo que foram produzidos nos anos 70, 80 e 90 do século passado. Alguns dos seus maiores expoentes encontram-se, por exemplo, no Reino Unido ${ }^{16-18}$ e na Suíça ${ }^{19,20}$. Deste tipo de pesquisa, e com base em modelos matemáticos estruturais e não-estruturais ${ }^{21}$, é possível obter um conjunto importante de parâmetros designados por parâmetros do salto pubertário ${ }^{22}$, de que a idade em que ocorre o PVA é o mais importante.

O PVA é um indicador maturacional de grande relevância na pesquisa em Educação Física e Esporte $^{23}$ com crianças e jovens. Com base na idade de ocorrência do seu PVA, é possível classifica-los em três categorias maturacionais: avançados (do inglês, advanced), atrasados (do inglês, late) ou "normomaturo" (do inglês, on time $)^{24}$. É reconhecido que as diferenças de desempenho motor de crianças e jovens em contexto escolar e esportivo estão relacionadas com a correspondente variação na velocidade do seu crescimento físico e maturação biológica ${ }^{15}$. Por exemplo, meninos e meninas avançados maturacionalmente têm desempenhos mais elevados que seus pares normomaturos ou com atraso maturacional ${ }^{25}$. Com base em procedimentos estatistico-matemáticos relativamente complexos ${ }^{6}$, é possível não só identificar o PVA mas também alinhar a velocidade das alterações do desempenho pela idade em que ocorre o PVA. Ainda que a pesquisa desta natureza seja relativamente escassa, os resultados mais "recentes" de Philippaerts et al. ${ }^{11}$ com rapazes futebolistas Belgas e o de YAGUE e DE LA Fuente ${ }^{12}$ com adolescentes Espanhóis de ambos os sexos são elucidativos da sua importância, a que se adiciona a possibilidade de identificar com base nesta metodologia aspetos relevantes da noção de prontidão motora ${ }^{26}$.

\section{Método}

\section{Amostra}

Os dados utilizados no presente estudo provém de uma pesquisa de natureza multivariada denominada Projeto Crescer com Saúde no Cariri ${ }^{34}$. A amostra foi constituída por crianças e adolescentes oriundos das três principais cidades do Cariri: Juazeiro
No Brasil, os estudos longitudinais sobre o crescimento da altura são raros. Conseguimos localizar apenas dois: o primeiro refere-se a um estudo longitudinal-misto realizado em 1970, em Ilhabela, litoral norte de São Paulo. Com base numa amostra de 44 crianças (26 meninos e 18 meninas) acompanhadas durante cerca de 5 anos, DuARTE ${ }^{27}$ descreveu os parâmetros do PVA e a sua relação com o desempenho motor. $\mathrm{O}$ segundo foi conduzido no âmbito do Projeto Esporte Brasil (PROESP) por BERGMANN et al. ${ }^{28}$ tendo acompanhado durante 4 anos um grupo de 70 escolares de ambos os sexos de uma escola privada da cidade de Canoas/RS. O objetivo principal foi identificar o timing e a magnitude do PVA e do pico de velocidade do peso. Enquanto o primeiro utilizou o modelo matemático proposto por Preece e Baines ${ }^{29}$, o segundo recorreu a subtração de valores adjacentes de altura e dividiu o resultado pela diferença de idades, i.e., o método dos incrementos.

Os anos 70, 80 e 90 do século passado foram férteis no desenvolvimento matemático-estatístico de modelos para descrever o crescimento estatural com base em informação longitudinal. Estão disponíveis, para além do modelo de Preece e Baines ${ }^{29}$, o modelo ICP de Karlberg ${ }^{30}$, o de Kanefuji e Shohoji ${ }^{31}$, o $\mathrm{JPA}_{2}{ }^{32}$ e o modelo triplo logístico proposto por Bock et al. ${ }^{33}$. O presente estudo, aproveitando esta vasta teia de conhecimentos, pretende contribuir com novas informaçôes relativas ao crescimento de crianças e jovens Brasileiros, sobretudo da Região Nordeste. Os seus objetivos são os seguintes: (1) apresentar as curvas médias da velocidade da altura de crianças e adolescentes de ambos os sexos da Região do Cariri Cearense; (2) estimar as idades em que ocorrem os parâmetros do salto pubertário; (3) comparar os valores obtidos com referências nacionais e internacionais, bem como (4) sugerir o uso do modelo logístico triplo proposto por Bock et al..$^{33}$ quando a informação disponível é de natureza longitudinal-mista.

do Norte, Crato e Barbalha. O estudo teve um delineamento longitudinal-misto com quatro coortes, sobreponíveis, constituídas por 100 crianças e adolescentes de ambos os sexos em cada coorte (TABELA 1). No entanto, para este estudo, foram selecionadas um total de 280 crianças e adolescentes de ambos os sexos que tinham participado, no 
mínimo, em 4 das 6 avaliações realizadas no decorrer dos três anos da recolha. A TABELA 2 apresenta a distribuição da amostra por sexo, coorte e idade.
O projeto Crescer com Saúde no Cariri foi aprovado pelo Comitê de Ética em Pesquisa da Faculdade de Medicina de Juazeiro do Norte (CEP/ FMJ $-n^{\circ}$ 01/07).

TABELA 1 - Coortes e distribuição das idades em que foram efetuadas as avaliações nos três anos do estudo.

\begin{tabular}{lllllllllllllll}
\hline \multicolumn{10}{c}{ Organizaçáo das coortes - Avaliaçóes semestrais } \\
Idades (em anos)
\end{tabular}

TABELA 2 - Frequências de casos e idades por coorte e sexo.

\begin{tabular}{cccc}
\hline Coortes & Idades (anos) & Fem & Masc \\
\hline C1 & $(8-10)$ & 46 & 44 \\
\hline C2 & $(10-12)$ & 38 & 40 \\
\hline C3 & $(12-14)$ & 37 & 45 \\
\hline C4 & $(14-16)$ & 15 & 15 \\
\hline Total & & 136 & 144 \\
\hline
\end{tabular}

Equipe de campo e processo de treino

Em função do extenso processo de recolha da informaçáo do projeto anteriormente mencionado, e por forma a minimizar os erros de medição durante todo o período de avaliação, foi constituída uma equipe de trabalho devidamente treinada para a aplicação exaustiva dos protocolos de medição. A equipe de campo foi composta por alunos e professores dos cursos de Licenciatura em Educação Física da Universidade Regional do Cariri - URCA e IFCE. Os pesquisadores internacionais principais do projeto são provenientes da Universidade do Porto, Portugal, e Katholieke Universiteit Leuven, Bélgica, que treinaram toda a equipe e supervisionaram um estudo piloto realizado numa amostra de 25 crianças $^{34}$.

\section{Protocolo de medição}

Todas as medições foram efetuadas segundo os protocolos descritos por Claessens et al..$^{35} \mathrm{~A}$ altura foi medida entre o vértex e o plano de referência do solo, com a cabeça na posição de Frankfurt, utilizando um estadiômetro portátil (Cardiomed ${ }^{\circledR}$ Welmy mod. 220).

\section{Controle e qualidade da informaçáo}

O controle da qualidade das mediçóes foi feita através de um estudo de confiabilidade, sendo que cinco crianças foram aleatoriamente reavaliadas a cada dia de coleta de dados. O erro técnico de medida da altura foi de $0.5 \mathrm{~cm}$, muito semelhante ao que ocorre noutros estudos internacionais ${ }^{36}$.

\section{Definição dos grupos de idade}

A idade decimal foi calculada em referência à data de nascimento de cada criança e a data da coleta dos dados. Os grupos de idade foram constituídos considerando a idade inferior em 0.50 e a idade superior 0.49 , ou seja, a idade intermediária é considerada como o ano completo. Assim, as crianças com 7.50 a 8.49 anos de idade inserem-se no grupo dos 8 anos.

\section{Modelaçáo das curvas de velocidade do crescimento}

O modelo triplo logístico foi inicialmente desenvolvido por Bock e THIssen ${ }^{37}$ e mais tarde 
aperfeiçoado por Bock et al. ${ }^{33,38}$. Este modelo é estruturado por três processos, primeira infância, segunda infância e adolescência que são descritos por três funçôes logísticas cuja adição traça o decurso do crescimento desde os seis meses de idade até à maturidade permitindo a presença de um salto pré-pubertário. Os seus oito parâmetros dão-lhe flexibilidade suficiente para reproduzir os valores originais da altura. A forma funcional do modelo é a seguinte:

$$
y=\frac{a_{1}}{\left[1+\exp \left(-b_{1} x-c_{1}\right)\right]^{d_{1}}}+\frac{a_{2}}{\left[1+\exp \left(-b_{2} x-c_{2}\right)\right]^{d_{2}}}+\frac{a_{3}}{\left[1+\exp \left(-b_{3} x-c_{3}\right)\right]^{d_{3}}}
$$

em que $\mathrm{y}=$ altura e $\mathrm{x}=$ idade, o termo $\mathrm{c}_{1}$ e os expoentes $\mathrm{d}_{1}, \mathrm{~d}_{2}$ e $\mathrm{d}_{3}$ não são estimados mas são constantes escolhidas de tal modo que garantem o melhor ajustamento das curvas. A escolha destas constantes foi obtida dos dados das pesquisas de Fels e de Berkeley, e são, respetivamente: 0.25, 0.815, 070 e 0.615 para meninos e $0.25,0.85,0.63$ e 0.60 para meninas ${ }^{33,38}$. A estimação dos parâmetros do modelo decorre em dois passos. No passo 1 é utilizado o método de máxima verosimilhança e no passo 2 recorre-se à estimação modal de Bayes, e os resíduos são suavizados com uma transformada de Fourier. Dado que o modelo assume três fases do crescimento estima, com grande precisão, os seis parâmetros do salto pubertário (idade em que se inicia o take-off, a velocidade no take-off e o valor estatural no take-off, a que se juntam a idade no PVA, ao PVA e a altura no PVA). Os cálculos e as representações gráficas das curvas foram obtidos no software AUXAL v. $3^{38}$.

\section{Resultados}

A TABELA 3 contém as estimativas dos seis parâmetros do salto pubertário: idade no início do salto (take-off), altura e velocidade instantânea no take-off, a idade de ocorrência do PVA, altura alcançada no PVA e a velocidade instantânea no PVA. Tal como descrito na literatura, a idade de ocorrência dos parâmetros do salto pubertário nas meninas acontece, em média, aproximadamente 2 anos mais cedo do que nos meninos. Nas meninas o take-off ocorreu, em média, aos 8.8 anos e nos meninos aos 10.3 anos. A altura alcançada foi de $127.3 \mathrm{~cm}$ nas meninas e de 134.1 nos meninos e a velocidade foi de $4.7 \mathrm{~cm}$ nos dois sexos. O PVA ocorreu, em média, aos 11.6 nas meninas e 13.6 nos meninos. Por seu lado, a altura no PVA foi de 143.1 nas meninas e de 154.9 nos meninos. Em média, a velocidade de crescimento foi de $7.1 \mathrm{~cm} /$ ano e $8.7 \mathrm{~cm} /$ ano nas meninas e meninos, respectivamente.

TABELA 3 - Valores médios ( \pm desvio-padrão) das estimativas dos parâmetros do salto pubertário da altura de meninas e meninos.

\begin{tabular}{lcc}
\hline $\begin{array}{c}\text { Salto pubertário } \\
\text { Parâmetros }\end{array}$ & $\begin{array}{c}\text { Meninas } \\
\mathbf{M} \pm \mathbf{d p}\end{array}$ & $\begin{array}{c}\text { Meninos } \\
\mathbf{M} \pm \mathbf{d p}\end{array}$ \\
\hline Take-Off & & \\
\hline Idade (anos) & $8.81 \pm 0.82$ & $10.32 \pm 1.14$ \\
\hline Altura $(\mathrm{cm})$ & $127.33 \pm 6.97$ & $134.06 \pm 8.60$ \\
\hline Velocidade (cm/ano) & $4.73 \pm 0.65$ & $4.68 \pm 0.75$ \\
\hline Pico de velocidade da altura & & $13.64 \pm 0.86$ \\
\hline Idade (anos) & $11.64 \pm 0.91$ & $154.85 \pm 5.53$ \\
\hline Altura $(\mathrm{cm})$ & $143.52 \pm 5.63$ & $8.72 \pm 1.09$ \\
\hline Velocidade $(\mathrm{cm} / \mathrm{ano})$ & $7.13 \pm 0.89$ & \\
\hline
\end{tabular}


Nas FIGURAS 1 e 2 estão representadas as curvas médias da velocidade, bem como a da distância, das meninas e meninos Caririenses. Os valores percentílicos da distância e da velocidade sobre os quais estão representadas as curvas médias dos e das Caririenses são os referidos por Hamill et al. ${ }^{39}$ e TANner e Davies ${ }^{40}$, respectivamente, para crianças e jovens Norte-Americanos; estas curvas são representadas automaticamente pelo software AUXAL.

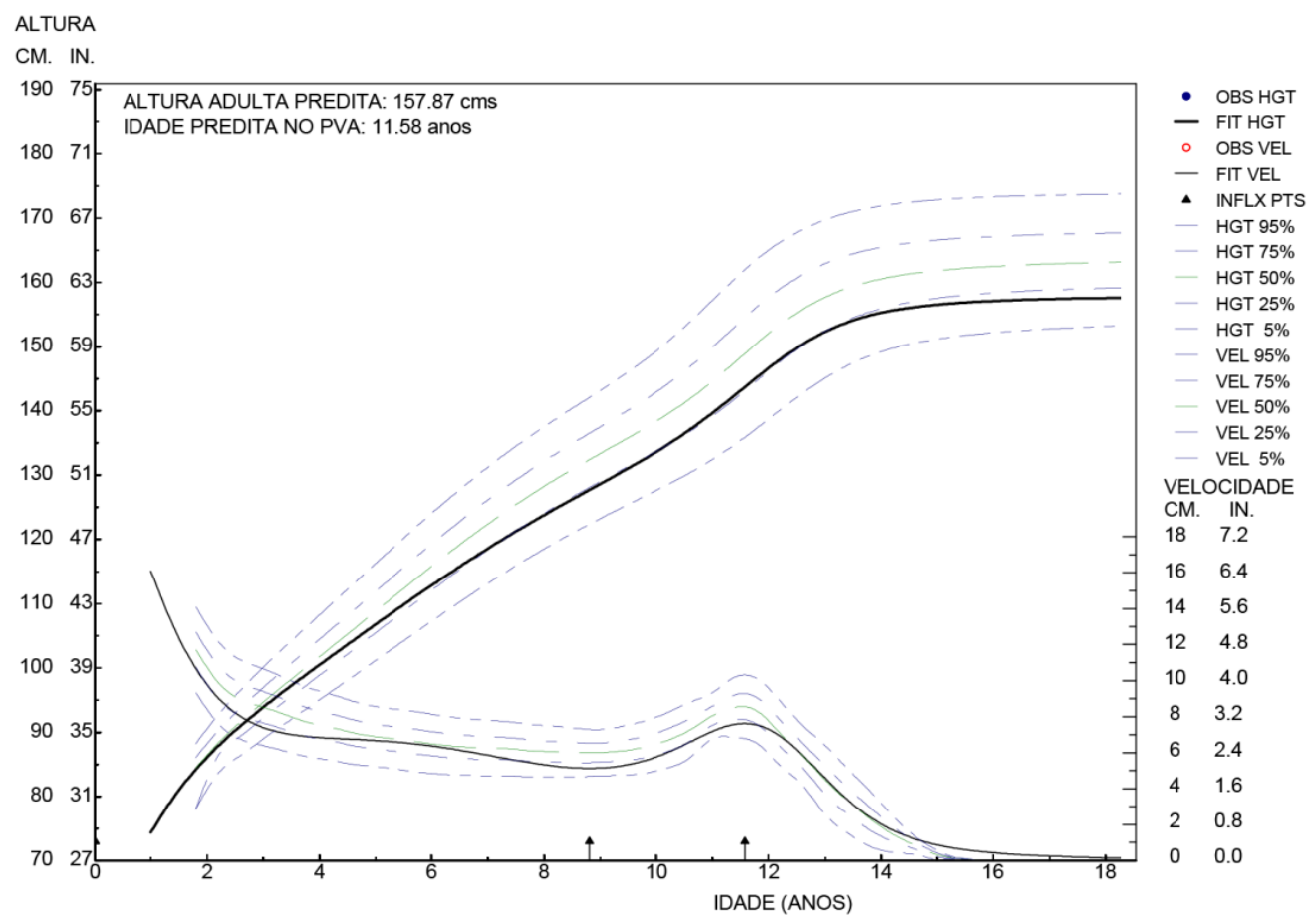

FIGURA 1 - Curvas de distância e velocidade da altura das meninas.

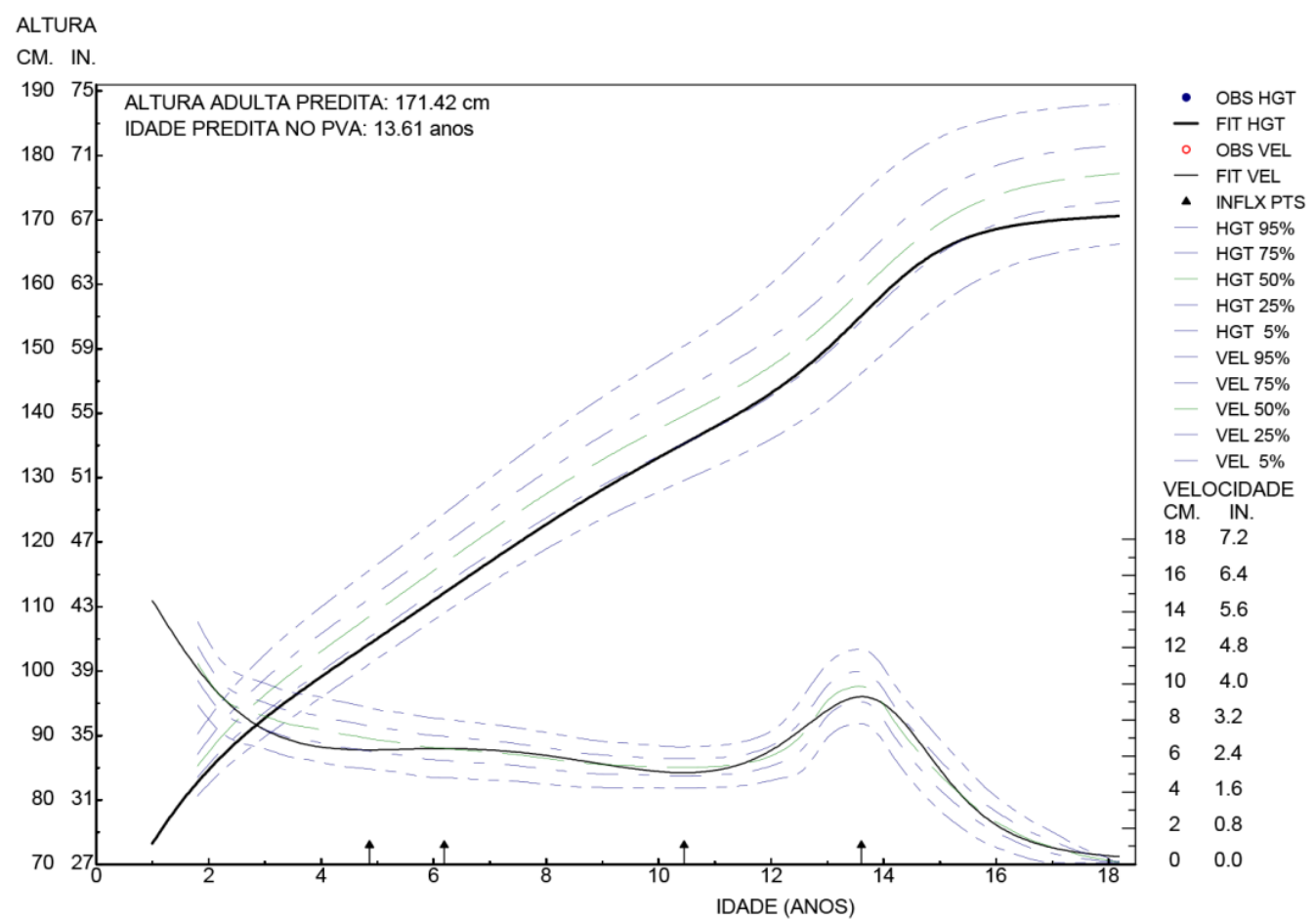

FIGURA 2 - Curvas de distância e velocidade da altura dos meninos. 
Os valores dos parâmetros do salto pubertário da presente pesquisa, bem como de outras referências nacionais e internacionais são apresentados na TABELA 4. Constata-se uma variação nos resultados das meninas e meninos. Por exemplo, nas meninas, a idade média mais baixa do PVA ocorreu aos 10.3 anos em crianças Japonesas ${ }^{41} \mathrm{e}$ a mais alta aconteceu aos 12.5 em crianças Americanas ${ }^{42}$. Nos meninos, a idade média mais baixa do PVA aconteceu aos 12.2 anos em Japoneses ${ }^{41}$ e a mais alta ocorreu aos 14.3 anos em meninos Indianos ${ }^{43}$. A velocidade de crescimento da altura mais elevada foi $9.1 \mathrm{~cm} /$ ano nas meninas Brasileiras, no estudo de São Paulo, enquanto que a mais baixa ocorreu nas meninas Suíças $(7.1 \mathrm{~cm} / \mathrm{ano})$. Entre os meninos, a velocidade mais elevada foi de $11.8 \mathrm{~cm} / \mathrm{ano}^{41}$ e a mais baixa foi de $7.3 \mathrm{~cm} / \mathrm{ano}^{44}$, ambas em estudos Japoneses. $\mathrm{Na}$ mesma tabela são também reportadas as diferenças $(\Delta)$ da idade de ocorrência do PVA e da velocidade do PVA entre as pesquisas realizadas dos diferentes países e as do presente estudo. $\mathrm{Na}$ idade no PVA das meninas a maior diferença $(-1.3$ anos) foi constatada relativamente ao estudo desenvolvido no Japão ${ }^{41}$ e a mais baixa (-0.1 ano) relativamente à amostra de Ilhabela ${ }^{27}$. Nos meninos, a maior diferença ( -1.4 anos) foi encontrada relativamente à pesquisa de Fujir e MatsuUra ${ }^{41}$ e a menor (0.2 anos) relativamente às reportadas por $\operatorname{LEE}^{42}$ e BuRns et al. ${ }^{45}$ em crianças e jovens Norte-Americanas e Canadenses, respectivamente. As diferenças no PVA variaram entre 0.0 e $2.1 \mathrm{~cm} /$ ano (Suíça e Japáo, respectivamente) nas meninas, e nos meninos variaram entre 0.0 e 1.7 cm/ano (India e Canadá, respectivamente).
${ }^{*}$ Os valores de $\Delta$ foram calculados com base nos dados de cada pesquisa relativamente aos do presente estudo.

TABELA 4 - Quadro comparativo dos diferentes estudos, países, métodos, idades de ocorrência do PVA, velocidade no PVA de meninas e meninos e diferenças $(\Delta)$ de resultados relativamente ao presente estudo.

\begin{tabular}{|c|c|c|c|c|c|c|c|c|c|c|}
\hline \multirow[b]{2}{*}{ País } & \multirow[b]{2}{*}{ Referência } & \multirow[b]{2}{*}{ Método } & \multicolumn{4}{|c|}{ Meninas } & \multicolumn{4}{|c|}{ Meninos } \\
\hline & & & $\begin{array}{c}\text { PVA } \\
\text { Idade }\end{array}$ & $\begin{array}{c}\Delta^{*} \\
\text { ano }\end{array}$ & $\begin{array}{c}\text { PVA } \\
\text { cm/ano }\end{array}$ & $\begin{array}{c}\Delta^{*} \\
\text { cm/ano }\end{array}$ & $\begin{array}{c}\text { PVA } \\
\text { Idade }\end{array}$ & $\begin{array}{c}\Delta^{*} \\
\text { ano }\end{array}$ & $\begin{array}{c}\text { PVA } \\
\text { cm/ano }\end{array}$ & $\begin{array}{c}\Delta^{*} \\
\text { cm/ano }\end{array}$ \\
\hline USA & Lee (1980) & Preece-Baines & 12.5 & 0.9 & - & - & 13.8 & 0.2 & - & - \\
\hline Canadá & $\begin{array}{l}\text { Burns et al. } \\
(2001)\end{array}$ & Cubic Splines & 11.8 & 0.2 & 8.6 & 1.5 & 13.4 & -0.2 & 10.4 & 1.7 \\
\hline Inglaterra & $\begin{array}{l}\text { Tanner et al. } \\
(1976)\end{array}$ & Preece-Baines & 11.9 & 0.3 & 8.1 & 1.0 & 13.9 & 0.3 & 8.8 & 0.1 \\
\hline Suíça & $\begin{array}{l}\text { Largo } \\
\& \text { Prader } \\
(1983) \\
\end{array}$ & Preece-Baines & 12.2 & 0.6 & 7.1 & 0 & 13.9 & 0.3 & 9.0 & 0.3 \\
\hline Inglaterra & $\begin{array}{l}\text { Buckler } \\
\text { \&Wild } \\
(1987)\end{array}$ & Interpolation & 12.0 & 0.4 & 8.1 & 1.0 & 14.1 & 0.5 & 9.8 & 1.1 \\
\hline India & $\begin{array}{l}\text { Hauspie } \\
\text { et al. (1980) }\end{array}$ & Preece-Baines & 12.4 & 0.8 & 7.2 & 0.1 & 14.3 & 0.7 & 8.7 & 0 \\
\hline Japão & $\begin{array}{l}\text { Fujii \& } \\
\text { Matsuura } \\
(1999) \\
\end{array}$ & Wavelets & 10.3 & -1.3 & 9.0 & 2.1 & 12.2 & 1.4 & 9.0 & 0.3 \\
\hline Japão & $\begin{array}{l}\text { Nariyama } \\
\text { et al. }(2001)\end{array}$ & Preece-Baines & - & - & - & - & 13.0 & -0.6 & 9.3 & 0.6 \\
\hline Japão & $\begin{array}{l}\text { Kimura } \\
\text { et al. }(2003)\end{array}$ & Eye-ftting & 11.2 & 0.4 & 8.7 & 1.6 & 13.0 & -0.6 & 7.3 & -1.4 \\
\hline São Paulo & $\begin{array}{l}\text { Duarte } \\
(1993) \\
\end{array}$ & Preece-Baines & 11.5 & -0.1 & 9.1 & 2.0 & 13.9 & 0.3 & 9.7 & 1.0 \\
\hline RGS & $\begin{array}{l}\text { Bergmann } \\
\text { et al. }(2008)\end{array}$ & Dif. De Médias & 10.5 & -1.1 & 7.3 & 0.2 & 12.5 & -1.1 & 7.8 & -1.0 \\
\hline Ceará & $\begin{array}{l}\text { Presente } \\
\text { estudo }\end{array}$ & Triplo logístico & 11.6 & - & 7.1 & - & 13.6 & - & 8.7 & - \\
\hline
\end{tabular}




\section{Discussão}

A pesquisa sobre a dinâmica do crescimento físico oriunda de delineamentos longitudinais-puros, cujo propósito fundamental é produzir curvas de velocidade e a inventariaçáo dos valores dos seus parâmetros fundamentais, é pouco frequente no Brasil bem como noutros países muito provavelmente porque a sua conceção, estrutura operativa e financiamento arrastam problemas sérios na sua implementação $0^{46}$. A alternativa viável, de natureza longitudinal-mista, constituída por múltiplas coortes com pontos de sobreposição torna todo o processo mais célere, de implementação mais eficiente e de custos mais reduzidos $^{47}$. Foi com base nestes fatos que o Projeto Crescer com Saúde no Cariri ${ }^{34}$ foi concebido e realizado, o que permitiu construir curvas médias locais da velocidade, e curvas da distância, da altura de crianças e adolescentes ao mesmo tempo que se estimaram as idades em que ocorreram os parâmetros do salto pubertário.

No presente estudo, as idades no take-off ocorreram aos 8.8 e 10.3 anos, a altura alcançada foi de $127.3 \mathrm{~cm}$ e $134.0 \mathrm{~cm}$, com uma velocidade instantânea de $4.7 \mathrm{~cm} /$ ano em meninas e meninos, respectivamente. Esta diferença entre sexos é bem conhecida na literatura uma vez que o salto pubertário ocorre mais cedo nas meninas ${ }^{15}$. Não obstante o número reduzido das pesquisas que localizamos (ver TABELA 4) terem reportado estes resultados, há semelhanças com os valores referidos por Duarte em crianças de Ilhabela e com as crianças Indianas do estudo de Hauspie et al. ${ }^{43}$ Esta similitude nos valores, apesar da diferença nos anos das pesquisas, -17 anos para Duarte e -30 anos para Hauspie, as diferenças étnicas (crianças Brasileiras e Indianas), bem como o método de estimação dos parâmetros (modelo de Preece-Baines versus modelo Logístico triplo), sugere semelhança etária neste importante evento responsável pela emergência do salto pubertário.

O PVA nas meninas Caririenses ocorreu, em média, aos 11.6 anos e nos meninos aos 13.6 anos. Estes resultados estão em consonância com a literatura, dado que as meninas experimentam o início do salto pubertário em média, dois anos antes dos meninos; consequentemente atingem o PVA mais cedo ${ }^{15}$. A altura média alcançada foi de $143.5 \mathrm{~cm}$ e $154.8 \mathrm{~cm}$ e o PVA foi de $7.1 \mathrm{~cm} /$ ano e $8.7 \mathrm{~cm} /$ ano em meninas e meninos, respectivamente. Também aqui há semelhanças com as crianças de Ilhabela uma vez que as idades no PVA foram de 11.5 anos e 13.9 anos, bem como a altura média alcançada ( $146.2 \mathrm{~cm}$ e $159.6 \mathrm{~cm}$ ); em contrapartida a velocidade foi ligeiramente superior, $9.1 \mathrm{~cm} /$ ano e $9.7 \mathrm{~cm} /$ ano em meninas e meninos, respectivamente. Os resultados do Cariri foram semelhantes aos de crianças e jovens Inglesas reportados por BUCKLER e $\mathrm{WILD}^{17}, 12.0$ e 14.1 anos, bem como em TANNER et al. ${ }^{48}, 11.9$ nas meninas e aos 13.9 anos nos meninos. Por outro lado, os valores de Bergmann et al. ${ }^{28}$ das crianças e adolescentes oriundos de uma escola privada de Canoas, RS, são inferiores. É nossa convicção que diferenças na seleção das amostras e sua dimensão, bem como os métodos de estimação dos parâmetros do salto pubertário possam explicar algumas das diferenças encontradas, sobretudo relativamente a esta última pesquisa.

Não obstante o padráo do crescimento físico humano ser semelhante em qualquer parte do mundo, os valores alcançados em cada idade são bem diferentes como salientaram EvELETH e TANNER $^{3}$. Conforme se observa nas FIGURAS 1 e 2, os traçados das curvas médias da velocidade e da distância das crianças e jovens Caririenses foram sobrepostas às referências Norte-Americanas. A localização dos Caririenses está no percentil 25 destas referências. É importante ressaltar que estas e outras diferenças existentes entre a Região do Cariri e dos grupos populacionais referenciados nas pesquisas utilizadas nas comparaçóes se devem, também, a aspectos socioeconômicos, demográficos, ambientais e culturais. Por exemplo, as cidades da região do Cariri onde esta pesquisa foi realizada (Juazeiro do Norte, Crato e Barbalha) possuem índices de desenvolvimento humano classificados como baixo-a-moderado ${ }^{49}$. Há, também, alguma disparidade no nível socioeconômico das crianças da própria Região do Cariri, sendo que as crianças de escolas privadas possuem um melhor nível socioeconômico e são sistematicamente mais altas. Aos 17 anos, a diferença está compreendida entre 2.7 e $5.5 \mathrm{~cm}$ nas meninas e entre 2.1 a $4.1 \mathrm{~cm}$ nos meninos ${ }^{10}$; um padrão semelhante ocorre relativamente às estaturas das crianças e jovens Norte-Americanos tal como referido anteriormente por Silva et al. ${ }^{10}$

A modelação das séries de valores da altura continua a ser um desafio para auxologistas e matemáticos, não obstante estarem disponíveis diferentes modelos estruturais e não-estruturais que a resolvem satisfatoriamente. Exemplos deste 
esforço são, por exemplo, o JPA2 $2^{32}$, o BTT ${ }^{38}$ ou o Kernel Smoothing ${ }^{50}$. Ora o modelo triplo logístico usado nesta pesquisa, também conhecido por modelo BTT, é extremamente flexível para ser implementado em dados incompletos ou irregulares (casos de informação omissa ou em delineamentos longitudinais-mistos), bem como em situaçóes de crescimento atípico. Adicionalmente, Bock ${ }^{51}$ reportou vantagens no uso do modelo BTT relativamente aos modelos de Kanefuj e Shohoji ${ }^{31}$ e JPA2 ${ }^{32}$, salientou o fato de representar o salto pré-pubertário somente visível nos modelos nãoparamétricos, e mostrou ${ }^{38}$ a eficiência do modelo logístico triplo mesmo na ausência de informação completa face ao fato do crescimento físico ser extremamente regular e previsível.

A literatura tem reportado sistematicamente, uma tendência secular positiva no aumento da altura média não só nos países industrializados ${ }^{52}$, mas também nos países emergentes ${ }^{53}$ e em desenvolvimento ${ }^{54}$. $\mathrm{O}$ mesmo ocorre nos valores estaturais médios ${ }^{15}$, nas taxas de crescimento ${ }^{1}$ e no timing maturacional ${ }^{15}$. Estas mudanças são habitualmente associadas às melhorias na urbanização, redes sanitárias, cuidados primários de saúde e hábitos nutricionais ${ }^{55}$.

É importante salientar aspectos relevantes dos resultados do nosso estudo, sobretudo a sua utilização no melhor entendimento do desempenho motor. Por exemplo, depois de Philippaerts et al. ${ }^{11}$ terem estimado a idade no PVA de 232 adolescentes futebolistas Belgas alinharam a velocidade das alteraçóes do seu desempenho motor em conformidade. No teste de flexibilidade do tronco (trunk lift) o pico do desempenho ocorreu um ano após o PVA; por outro lado, no teste de força explosiva de membros superiores (standing long jump), e na prova de velocidade/agilidade (shuttle run $10 X 5 \mathrm{~m}$ ) os seus picos foram coincidentes com o PVA. No estudo de Yague e de la Fuente ${ }^{12}$ com 509 rapazes e 453 meninas Espanholas verificou-se que nas meninas o pico do seu desempenho motor na prova de resistência cardiorrespiratória (6 minute run) ocorreu 1 ano antes do PVA, enquanto que nos rapazes ocorreu um ano após o PVA. Por outro lado, nos testes de agilidade/velocidade (shuttle run $4 X 6 \mathrm{~m}$ ) e força e resistência abdominal (sit ups 30 seconds) ocorreu antes do PVA em ambos os sexos. $\mathrm{Na}$ pesquisa com crianças e adolescentes Brasileiros de Ilhabela Duarte ${ }^{27}$ verificou que nas meninas, nos testes de força explosiva de membros inferiores (standing long jump) e força de preensão manual (hand grip) ocorreram dois picos de desempenho, um 6 meses antes e outro 6 meses após o PVA; no teste de agilidade/velocidade (shuttle run) o pico do desempenho ocorreu 6 meses antes do PVA. Nos rapazes, o pico de desempenho no teste standing long jump ocorreu 6 meses antes do PVA, no teste de hand grip o pico do desempenho foi coincidente com o PVA, no teste de shuttle run ocorreu 6 meses antes do PVA, seguido de um novo aumento e depois um decréscimo no desempenho após o PVA. Em suma, esta informação é de grande importância em termos de identificação de momentos ótimos de velocidade do desempenho motor de meninos e meninas que pode ser de útil para os profissionais de Educação Física e Esporte na interpretação diferencial do desempenho dos seus alunos e atletas em resposta ao treino-instruçáo qualquer que seja o seu contexto.

O presente estudo contém, pelo menos, duas limitações que devem ser reconhecidas: (1) a náo apresentação de curvas individuais face ao número limitado de observaçôes por sujeito em cada coorte, bem como (2) o número reduzido de adolescentes na coorte 4 que está associado, de certo modo, à evasão escolar principalmente a partir dos 15 anos de idade nas escolas públicas da região. Para além de ser conhecida a robustez do modelo logístico triplo em lidar de modo eficiente em situaçóes desta natureza ${ }^{38,51}$, foram geradas curvas individuais bem como informação dos parâmetros do salto para cada criança/adolescente mas que náo foram apresentadas neste artigo dada a sua extensão. Apesar destas limitações, também há que destacar alguns pontos fortes: (1) o delineamento longitudinal-misto da pesquisa bem como a amplitude etária de crianças e adolescentes amostrados; (2) o uso de um dos mais eficientes modelos matemático-estatísticos para modelar curvas da velocidade do crescimento físico mesmo na presença de informação omissa; (3) a disponibilização de informação sobre os parâmetros do salto pubertário de crianças e adolescentes Caririenses, que é bem escassa no Brasil.

Em suma, conclui-se que as curvas da velocidade de crescimento da altura das crianças do Cariri mostram um padrão similar ao observado em estudos de diferentes países. Apesar da defasagem temporal entre o presente estudo e os demais utilizados nas comparaçôes, as idades de ocorrência do PVA, bem como os valores encontrados nos parâmetros do salto pubertário são muito semelhantes daqueles verificados em pesquisas nacionais e internacionais. 


\section{Abstract}

Height velocity curves and adolescent growth spurt parameters of children and adolescents from Cariri

We present the distance and velocity curves of height, ages that occurred growth parameters spurt and comparing the values obtained with national and international references. The sample includes 280 children and adolescents of both sexes who participated in the project Healthy Growth in Cariri Study. We used the triple logistic model implemented in AUXAL software. Ages in take-off occurred at 8.8 and 10.3 years old, the height reached was 127.3 and $134.0 \mathrm{~cm}$, and the annual increments was $4.7 \mathrm{~cm}$ for both sexes.

The peak height velocity (PHV) occurred at 11.6 years in girls and 13.6 years in boys. The average height reached was 143.5 and $154.8 \mathrm{~cm}$ with an annual increments of 7.1 and $8.7 \mathrm{~cm} /$ year between girls and boys, respectively. The growth curves of the height of Cariri children shows a similar pattern to that observed in other studies. Despite the time lag between this study and the others used in the comparisons, the PHV occurrence of ages as well as the values found in the pubertal spurt parameters are similar.

KEYWORDS: Growth; Maturation; Children; Adolescents; Height.

\section{Agradecimentos}

Os autores agradecem aos órgãos de fomento no Brasil: CAPES - Coordenação de Aperfeiçoamento de Pessoal de Nível Superior e, em Portugal: FCT - Fundação Para a Ciência e a Tecnologia.

\section{Referências}

1. Stulp G, Barret L. Evolutionary perspectives in human heigth variation. Biol Rev Camb Philos Soc. 2016;91(1):206-34.

2. Allen HL, Estrada K, Lettre G, Berndt S, Weedon M, Rivadeneira F, et al. Hundreds of variants clustered in genomic loci and biological pathways affect human height. Nature. 2010;467(7317):832-8.

3. Eveleth PB, Tanner JM. Worldwide variation in human growth. 2a ed. Cambridge: Cambridge University Press; 1990.

4. Garza C, de Onis M. A new international growth reference for young children. Am J Clin Nutr. 1999;70(1):169-72.

5. Queiroz VA, Assis AM, Pinheiro SM, Ribeiro Junior HC. Predictors of linear growth in the first year of life of a prospective cohort of full term children with normal birth weight. J Pediatr. 2012;88(1):79-86.

6. Beunen G, Malina R, van't Hof M, Simons J, Ostyn M, Renson R, et al. Adolescent growth and motor performance: a longitudinal study in belgian boys. Illinois: Human Kinetics; 1988.

7. Chaves R, Baxter-Jones A, Souza M, Santos D, Maia J. Height, weight, body composition, and waist circumference references for 7- to 17-year-old children from rural Portugal. Homo. 2015;66(3):264-77.

8. de Souza M, Eisenmann J, Santos D, de Chaves R, Forjaz CM, Maia J. Modeling the dynamics of BMI changes during adolescence: the Oporto growth, health and performance study. Int J Obes. 2015;39(7):1063-9.

9. Freitas DL, Maia JA, Beunen G, Lefevre JA, Claessens AL, Marques AT, et al. Crescimento somático, maturação biológica, aptidão física, actividade física e estatuto sócio-econômico de crianças e adolescentes madeirenses: o estudo de crescimento da Madeira. Funchal: Universidade da Madeira; 2002.

10. Silva SP, Maia J, Claessens A, Beunen G, Pan H. Growth references for Brazilian children and adolescents: healthy growth in Cariri study. Ann Hum Biol. 2012;39(1):11-8.

11. Philippaerts RM, Vaeyens R, Janssens M, Renterghem BV, Matthys D, Craen R, et al. The relationship between peak height velocity and physical performance in youth soccer players. J Sport Sci. 2006;24(3):221-30.

12. Yague P, de la Fuente J. Changes in height and motor performance relative to peak height velocity: a mixed-longitudinal study of Spanish boys and girls. Am J Hum Biol. 1998;10(5):647-60. 
13. Kuczmarski R, Ogden C, Guo S, Grummer-Strawn L, Flegal K, Mei Z, et al. 2000 CDC growth charts for the United States: methods and development. Vital Health Stat. 2002;11(246):1-190.

14. Molinari L, Gasser T. The human growth curve: distance, velocity and acceleration. In: Hauspie R, Cameron N, Molinari L, editors. Methods in human growth research. Cambridge: Cambridge University Press; 2004. p. 27-53.

15. Malina R, Bouchard C, Bar-Or O. Growth, maturation and physical activity. 4a ed. Illinois: Human Kinetics Books; 2004.

16. Goldstein H. The design and analysis of longitudinal studies. London: Academic Press; 1979. 199 p.

17. Buckler JM, Wild J. Longitudinal study of height and weight at adolescence. Arch Dis Child. 1987;62(12):1224-32.

18. Tanner JM, Whitehouse RH. Clinical longitudinal standards for height, weigth, height velocity, weight velocity and stages of puberty. Arch Dis Child. 1976;51:170-9.

19. Largo R, Prader A. Pubertal development in Swiss boys. Helv Paediatr Acta. 1983;38(3):211-28.

20. Largo R, Prader A. Pubertal development in Swiss girls. Helv Paediatr Acta. 1983;38(3):229-43.

21. Gasser T, Gervini D, Molinari L. Kernel estimation, shape-invariant modelling and structural analysis. In: Hauspie R, Cameron N, Molinari L, editores. Methods in human growth research. Cambridge: Cambridge University Press; 2004.

22. Hauspie R. Adolescence: somatic growth and sex differences. In: Cameron N, editor. Human growth and development. London: Academic Press; 2002. p. 45-63.

23. Beunen G, Malina R. Growth and biologic maturation: relevance to athletic performance. In: Ebestreit $\mathrm{H}$, Bar-Or O, editores. The young athlete encyclopaedia of sports medicine. New Jersey: Blackwell; 2008. p. 3-17.

24. American Alliance for Health, Physical Educations, Recreation and Dance. AAHPERD health related physical fitness manual. Washington: AAHPERD; 1980.

25. Beunen G, Malina R. Growth and physical performance relative to the timing of the adolescent spurt. ESSR. 1988;16(1):503-40.

26. Lima MCAM. Prontidão desportivo-motora: um estudo na escola básica de Leça da Palmeira. Porto: Universidada do Porto; 2013.

27. Duarte MFS. Longitudinal study of pubertal peak height velocity and related morphological and functional components in Brazilian children. Illinois: University of Illinois; 1993.

28. Bergmann G, Bergmann M, Pinheiro E, Moreira R, Marques A, Gaya A. Estudo longitudinal do crescimento corporal de escolares de 10 a 14 anos: dimorfismo sexual e pico de velocidade. Rev Bras Cineantropom Desempenho Hum. 2008;10(3):249-54.

29. Preece MA, Baines MJ. A new family of mathematical models describing the human growth curve. Ann Hum Biol. 1978;5(1):1-24.

30. Karlberg J. A biologically-oriented mathematical model (ICP) for human growth. Acta Paediatr Scand Suppl. 1989;350:70-94.

31. Kanefuji K, Shohoji T. On a growth model of human height. 1990;54(4):155-65.

32. Jolicoeur P, Pontier J, Abidi H. Asymptotic models for the longitudinal growth of human stature. Am J Hum Biol. 1992;4(4):461-8.

33. Bock RD, du Toit SH, Thissen D. Auxal: auxological analysis of longitudinal measurements of human stature. Chicago: Scientific Software International; 1994.

34. Silva SP, Maia J, Beunen G. Crescer com saúde no Cariri: o estudo do crescimento físico e desempenho motor de crianças e jovens caririenses. Crato: RDS; 2014.160 p.

35. Claessens A, Beunen G, Malina R. Antropometry, physique, body composition, and maturity. In: Armstrong N, Mechelen WV, editores. Paediatric exercise science and medicine. 2a ed. New York: Oxford University Press; 2008.

36. Ulijaszek SJ, Lourie JA. Intra- and inter-observer error in anthropometric measurement. In: Ulijaszek SJ, Mascie-Taylor CG, editores. Anthropometry: the individual and the population. Cambridge: Cambridge University Press; 1994. p. 30-55.

37. Bock RD, Thissen DM. Fitting multi-component models for growth in stature. 9th International Biometrics Conference; 1976 ago 22-7; Boston. Raleigh: Biometric Society; 1976. p. 431-42.

38. Bock RD, editor. Auxal: auxological analysis of longitudinal measurements of human stature. Lincolnwood: Scientific Software International; 2003.

39. Hamill PV, Driz TA, Jonhson CH, Reed RB, Roche AF, Moore WN. Physical growth: National Center for Health Statistics percentiles. Am J Clin Nutr. 1979;32(3):607-29.

40. Tanner JM, Davies PSW. Clinical longitudinal standards for height and weight velocity for North American children. J Pediatr. 1985;107(3):317-29.

41. Fujii K, Matsuura Y. Analysis of the velocity curve for height by the Wavelet interpolation method in children classified by maturity rate. Am J Hum Biol. 1999;11(1):13-30.

42. Lee PA. Normal ages of pubertal events among American males and females. J Adolesc Health Care. 1980;1(1):26-9. 
43. Hauspie RC, Das SR, Preece MA, Tanner JM. A longitudinal study of the growth in height of boys and girls of West Bengal (India) aged 6 months to 20 years. Ann Hum Biol. 1980;7(5):429-41.

44. Kimura J, Tachibana K, Imaizumi K, Kurosawa K, Kuroki Y. Longitudinal growth and height velocity of Japanese children with Down's syndrome. Acta Paediatr. 2003;92:1039-42.

45. Burns SJ, Mirwald RL, Bailey DA. Timing and magnitude of peak height velocity and peak tissue velocities for early, average and late maturing boys and girls. Am J Hum Biol. 2001;13(1):18.

46. van Mechelen W, Mellenberg GJ. Problems and solutions in longitudinal research: from theory to practice. Int J Sports Med. 1997;18(supl 3):S238-45.

47. Menard S. Longitudinal research. London: Sage University Papers; 2002.

48. Tanner JM, Whitehouse RH, Marubini E, Resele LF. The adolescent growth spurt of boys and girls of the Harpenden growth study. Ann Hum Biol. 1976;3(2):109-26.

49. Programa das Naçóes Unidas para o desenvolvimento 2015 [Homepage na Internet]. Genebra: PNUD; [citado em 2018 mar 14]. Disponível em: <https://goo.gl/oZWJce>.

50. Gasser T, Kohler W, Muller H-G, Kneip A, Largo R, Molinari L, et al. Velocity and acceleration of height growth using kernel estimation. Ann Hum Biol. 1984;11(5):397-411.

51. Bock DR. Growth models. In: Hauspie R, Lindgren G, Falkner F, editores. Essays on auxology presented to James Mourilyan Tanner by colleagues and fellows. Wenyn Garden City: Castlemead Publications; 1995. p. 28-38.

52. Malina RM. Research on secular trends in auxology. Anthropol Anz. 1990;48(3):209-27.

53. Vargas DM, Arena LF, Soncini AS. Tendência secular do crescimento em estatura em Blumenau Brasil e sua associaçáo com o índice de desenvolvimento humano (IDH ). Rev Assoc Med Bras. 2010;56(3):304-8.

54. Santos FK, Maia JA, Gomes TNQ, Daca T, Madeira A, Katzmarzyk PT, et al. Secular trends in growth and nutritional status of Mozambican school-aged children and adolescents. Plos One. 2014;9(12):1-15.

55. Quintana-Domeque C, Bozzoli C, Bosch M. The evolution of adult height across Spanish regions, 1950-1980: a new source of data. Econ Hum Biol. 2012;10(3):264-75.

\begin{tabular}{r|l} 
ENDEREÇO & \\
Simonete Silva & Recebido para publicação: 08/11/2015 \\
Rua Cel. António Luiz, 1.161 - Pimenta & Revisado: 06/01/2016 \\
63105-Ooo - Crato - CE - BRASIL & Aceito: 26/02/2016 \\
e-mail: simonete.silva@urca.br & \\
&
\end{tabular}

